\title{
Short-term Wind Prediction Using an Ensemble of Particle Swarm Optimised FIR Filters
}

\author{
J Dowell* $^{*}$ S Weiss ${ }^{\dagger}$ \\ ${ }^{*}$ Wind Energy Systems Centre for Doctoral Training, University of Strathclyde, Glasgow \\ ${ }^{\dagger}$ Department of Electronic and Electrical Engineering, University of Strathclyde, Glasgow \\ \{jethro.dowell,stephan.weiss\}@strath.ac.uk
}

\begin{abstract}
Due to the large and increasing penetration of wind power around the world, accurate power production forecasts are required to manage power systems and wind power plants. In this paper we propose an ensemble of particle swarm optimised filtering technique for 1-hour-ahead prediction of hourly mean wind speed and direction. The performance of the new method is assessed by testing it on data from 13 locations around the UK where it performs comparably to linear techniques but is able to provide significant improvement at a subset of locations.
\end{abstract}

\section{Introduction}

Short-term wind power prediction is of great value to energy traders and power system operators. However, wind power prediction is notoriously difficult due to the non-stationary and non-linear nature of wind, and has to rely on solutions that are sufficiently low in computational complexity in order to facilitate implementation as real-time solution [1], as opposed to more complex numerical weather prediction models with a greater forecast horizon which only update every several hours [2].

The non-stationarity is strongly linked to the annual cycle of seasons and as such has been approached in $[3,4]$ by developing a cyclo-stationary Wiener filter. Linear filters satisfy the constraint of low complexity [1] but have been found to be particularly limited by their delayed response to fast changes in wind regime, which occur due to changing weather patterns.

Therefore, in this submission we aim to investigate a prediction method that lifts the linear constraint in [4]. In particular, particle swarm optimisation has been applied to FIR filters for prediction [5]. The adaptive filters exhibit a good response to sudden changes in wind regime while also tracking the type of non-stationarities that have been identified for the linear case. Further more, an ensemble of particle swarm optimised FIR filters is found to produce the most consistent 1-hour-ahead prediction.

The wind model and particle swarm optimisation (PSO) algorithm are described in Sections 2. and 3.1, and the application of PSO to the wind model for prediction is detailed in Sections 3.2 and 3.3. Results from testing the proposed algorithm are presented and discussed in Section 4. before some conclusions and suggestions for future work are presented in 5 .

\section{Wind Model}

The hourly mean wind speed and direction at discrete time index $t$ are modelled as the magnitude and phase of a complex random variable, $y[t]$, which is the weighted linear combination of $N$ past measurements of $y[t]$ and some error of unknown statistics, $\varepsilon[t]$. The past measurements of $y[t]$ and the complex prediction coefficients, $w_{\tau}[t]$, are arranged as vectors $\mathbf{y}_{t}$ and $\mathbf{w}[t]$ of size $N$, respectively,

$$
y[t]=\sum_{\tau=1}^{N} w_{\tau}[t] y[t-\tau]+\varepsilon[t]=\mathbf{w}[t]^{\mathrm{T}} \mathbf{y}_{t}+\varepsilon[t]
$$

where the coefficients of $\mathbf{w}[t]$ form a time dependent FIR filter of length $N$, and $\cdot^{\mathrm{T}}$ denotes the transpose operator.

We choose $\mathbf{w}[t]$ to make a prediction, $\hat{y}[t]$, of $y[t]$ by minimising the prediction error $\varepsilon[t]$. The prediction problem can now be written thus:

$$
\begin{aligned}
& \hat{y}[t]=\mathbf{w}[t]^{\mathrm{T}} \mathbf{y}_{t}, \\
& \varepsilon[t]=y[t]-\hat{y}[t] .
\end{aligned}
$$

By making assumptions about the statistical properties of $\varepsilon[t]$, one could proceed to formulate a number of linear predictors for $y[t]$, however, it is our goal to proceed without making such assumptions.

\section{Prediction Based on Particle Swarm Optimisation}

\subsection{Review of Particle Swarm Optimisation}

The particle swarm optimisation (PSO) algorithm [5,6], is a powerful and intuitive tool inspired by the social behaviour of swarms in nature. A group of candidate solutions, or particles, are flown through a given problem space with their velocities influenced by both their own performance, evaluated by some 


\begin{tabular}{|c|c|}
\hline Parameter & Value \\
\hline$c_{0}$ & 1.5 \\
$c_{1}$ & 0.5 \\
$c_{2}$ & 0.5 \\
$v_{\max }$ & 0.05 \\
No. of Particles & 25 \\
Memory & 48 \\
Ensemble Size & 20 \\
\hline
\end{tabular}

Table 1. List of parameter values used in PSO algorithm.

cost function, and that of the most successful member of the swarm.

Particle accelerations are randomly perturbed to produce the swarm-like behaviour observed in nature and to allow for the problem space to be appropriately explored. The swarm is accelerated towards the best known minima of the cost function while continuously searching for a better solution.

Algorithm: The $i^{\text {th }}$ particle occupies the position $p_{i}(t)$ at time $t$ in a problem space governed by cost function $C(p)$, has velocity $v_{i}$, memory of its own previous best position, $p_{i, b e s t}$, and knowledge of the previous best position of any particle $p_{g, b e s t}$.

1. Initialise particles with random positions and velocities in the problem space for time step $t=0$. Assign $p_{i, b e s t}=$ $p_{i}(0)$ for all particles and set $p_{g, \text { best }}=\underset{p_{i, b e s t}}{\arg \min }\left(C\left(p_{i, \text { best }}\right)\right)$.

\section{Repeat:}

2. For each particle, calculate $C\left(p_{i}(t)\right)$. If $C\left(p_{i}(t)\right)<C\left(p_{i, b e s t}\right)$ then $p_{i, \text { best }}=p_{i}(t)$. If $C\left(p_{i}(t)\right)<C\left(p_{g, \text { best }}\right)$ then $p_{g, \text { best }}=$ $p_{i}(t)$.

3. Update velocity, $v_{i}(t)$, and position of each particle:

$$
\begin{gathered}
v_{i}(t+1)=c_{0} v_{i}(t)+r_{1} c_{1}\left(p_{i, \text { best }}-p_{i}(t)\right) \\
\quad+r_{2} c_{2}\left(p_{g, \text { best }}-p_{i}(t)\right), \\
p_{i}(t+1)=p_{i}(t)+v_{i}(t+1),
\end{gathered}
$$

where $r_{1}, r_{2} \sim U(0,1)$ are random weights, $c_{0}$ is the inertial weight, $c_{1}$ is the cognition acceleration, and $c_{2}$ is the social acceleration.

4. Advance one time step and return to step 2.

\subsection{PSO for FIR Prediction}

The algorithm described in Section 3.1 is applied as if in real time to the FIR predictor described by (2). Each particle in the swarm is a candidate for the FIR filter and at each time step the best performing particle is selected to make the next prediction. The problem space is therefore the $N$-dimensional complex space $\mathbb{C}^{N}$. Each particle, $p_{i}(t)$, and its associated velocity, $v_{i}(t)$, is a complex vector of length $N$.

The cost function to be minimised is the prediction error, $\varepsilon[t]$. When a new measurement is received, the potential past performance of all the particles can be evaluated and the best performing particle selected to make the next prediction.
In addition to the basic algorithm, a maximum particle speed, $v_{\max }$, is enforced to restrict the step size of particles in order to control the resolution of the optimization, akin to $[7,8]$. If a particle's speed exceeds $v_{\max }$, it is reduced to $v_{\max }$.

Since the wind signal is non-stationary, the optimal solution we are searching for is not static in the problem space $a$ priori the PSO must be adjusted to allow for out-of-date solutions to be forgotten. Therefore, the particles are given a finite memory of the previous best locations $p_{i, \text { best }}$ and $p_{g, \text { best }}$.

Finally, due to the stochastic nature of the PSO algorithm, the most consistent prediction is produced by generating an ensemble of FIR filters, each individual filter optimised by a separate particle swam, and taking the mean prediction to be the ensemble prediction. Therefore, we formulate an ensemble of particle swarm optimised FIR (EPSO-FIR) filters.

The $k^{\text {th }}$ member of the ensemble comprising $K$ members optimises $\mathbf{w}_{k}[t]$ to produce the prediction $\hat{y}_{k}[t]$, as in (2). The ensemble prediction, $\tilde{y}[t]$,

$$
\tilde{y}[t]=\frac{1}{K} \sum_{k=1}^{K} \hat{y}_{k}[t],
$$

is the mean of the individual members' predictions.

\subsection{Parameter Choice}

The parameters of the PSO have been chosen heuristically, after exhaustive tests, to produce appropriate swarm behaviour and to minimise the root mean-squared error over the prediction period. Table 1 details the parameter values.

The coefficients of the velocity equation are chosen to produce swarm-like behaviour to enable the PSO algorithm to function as intended. This requires a balance between cognition and social acceleration to maintain a healthy particle distribution, and a sufficiently large inertial weight to ensure that the problem space is adequately explored. The maximum velocity is chosen to limit the distance each particle can travel in a single time step.

Each particle is given a memory of 48 time steps, i.e. 48 hours, since this is the time scale that the weather systems which govern the wind regime move across the UK, and is therefore an important component scale related to the wind signal's nonstationarity. An ensemble of 20 particle swarm optimised filters is found to produce consistent performance with little to be gained from using a larger ensemble.

\section{Results}

In this section we apply the proposed method to wind measurements and attempt to make 1-hour-ahead forecasts. The performance of the ensemble of particle swarm optimised FIR filters (EPSO-FIR) is compared to the complex LMS algorithm (CLMS), [3,9], and a single channel cyclo-stationary Wiener filter (CsWF) described in [4]. 


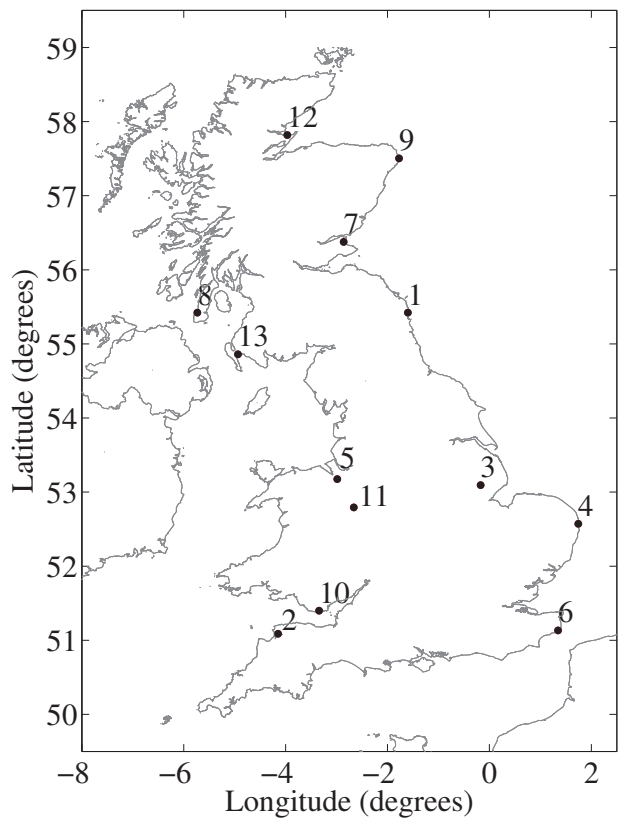

Figure 1. Locations of the 13 meteorological stations from which measurements have been used. Numbering corresponds to Table 3 .

All quoted errors are root mean-squared error (RMSE),

$$
\mathrm{RMSE}=\sqrt{\frac{1}{T} \sum_{t=1}^{T} \varepsilon[t] \varepsilon[t]^{*}},
$$

where the prediction error $\varepsilon[t]$ is the difference between the predicted and measured wind velocity, i.e. not wind speed or direction independently.

\subsection{Description of Data}

The proposed approach is tested on wind data provided by the British Atmospheric Data Centre, which comprises of measurements made over 1 year - from 00:00h on 1/3/1997 to 23:00h on 28/2/1998 - obtained from 13 sites across the UK detailed in Figure 1. The measurements are taken in open terrain at a height of $10 \mathrm{~m}$, and comprises hourly averages that are quantised to a $10^{\circ}$ angular granularity and integer multiples of one $\operatorname{knot}\left(0.515 \mathrm{~ms}^{-1}\right)$ [10].

\subsection{Prediction}

Some example time series from individual and ensemble predictions are illustrated in Figure 2. The individual filters are able to track large and fast changes in the wind speed well but do not do so consistently. This tracking is often accompanied by a significant over-shoot as the filter fails to anticipate the sudden change in gradient.

The inconsistent behaviour of the individual filters is lost when an ensemble of predictions is averaged, resulting in an over

\begin{tabular}{|c|c|c|}
\hline Site & $\begin{array}{c}\text { Individual } \\
\text { PSO-FIR }\end{array}$ & $\begin{array}{c}\text { Ensemble } \\
\text { PSO-FIR }\end{array}$ \\
\hline Boulmer & $2.0568-2.8172$ & 1.6347 \\
Cheivenor & $1.7105-2.3543$ & 1.3361 \\
Langdon Bay & $1.9056-2.4388$ & 1.6326 \\
Peterhead Harbour & $2.1990-2.9896$ & 1.8113 \\
Roose & $1.8201-2.1254$ & 1.5887 \\
\hline
\end{tabular}

Table 2. Comparison of the root mean-squared errors (RMSE) from individual predictors and the RMSE from the corresponding ensemble prediction.

\begin{tabular}{|c|c|c|c|c|}
\hline$\#$ & Site & CLMS & CsWF & EPSO-FIR \\
\hline 1 & Boulmer & 1.6252 & $\mathbf{1 . 6 2 3 8}$ & 1.6347 \\
2 & Chivenor & 1.7812 & 1.7790 & $\mathbf{1 . 3 3 6 1}$ \\
3 & Coningsby & 1.2939 & $\mathbf{1 . 2 9 3 2}$ & 1.3231 \\
4 & Gorleston & 1.6071 & $\mathbf{1 . 6 0 9 0}$ & 1.6462 \\
5 & Hawarden Airport & 1.5984 & $\mathbf{1 . 5 9 4 8}$ & 1.6401 \\
6 & Langdon Bay & 1.7399 & 1.7423 & $\mathbf{1 . 6 3 2 6}$ \\
7 & Leuchars & 1.5783 & $\mathbf{1 . 5 7 1 7}$ & 1.6026 \\
8 & Machrihanish & 2.0591 & $\mathbf{2 . 0 5 3 2}$ & 2.0945 \\
9 & Peterhead Harbour & $\mathbf{1 . 7 8 0 1}$ & n/a* & 1.8113 \\
10 & Rhoose & 1.7596 & 1.7578 & $\mathbf{1 . 5 8 8 7}$ \\
11 & Shawbury & 1.5326 & $\mathbf{1 . 5 3 1 4}$ & 1.5701 \\
12 & Tain Range & 2.0262 & $\mathbf{2 . 0 2 2 4}$ & 2.1034 \\
13 & West Freugh & $\mathbf{1 . 8 2 6 0}$ & 1.8289 & 1.8626 \\
\hline
\end{tabular}

Table 3. Comparison of 1 hour ahead root mean-squared prediction error for the complex LMS (CLMS) algorithm, cyclo-stationary Wiener filter (CsWF) and the ensemble of particle swarm optimised FIR filters (EPSO-FIR). The RMSE for the best performing method is highlighted in bold.

* Implementation of the CsWF was not possible for Peterhead Harbour due to insufficient training data.

all reduction in error but a systematic lag in response to large changes in wind speed.

The benefit of taking the mean prediction from an ensemble of PSO optimised predictors is significant. The RMSE, measured over the entire year of predictions, for the ensemble prediction is substantially lower than that for the individual predictors. Some examples are given in Table 2.

The results from the EPSO-FIR prediction and the two linear methods are listed in Table 3. The EPSO-FIR is out performed by the other two methods at 10 of the 13 locations by approximately $4 \%$, however, it performs substantially better than both the CLMS and CsWF at three sites with a $15 \%$ reduction in RMSE, notably the three most southerly sites in the data set, see Figure 1.

The results provide evidence that PSO can afford a significant performance advantage for at least some sites in the current setting of the method. Whether there are any anomalies in those three sites that favour PSO over our previous techniques is difficult to established based on only three sites, and will be the subject of future investigation.

The behaviour of the particle swarm is good, the distribution of particles is such that a sensible region of the problem space is explored. The algorithm converges quickly and tracks the non-stationary wind signal well. The density evolution of the 


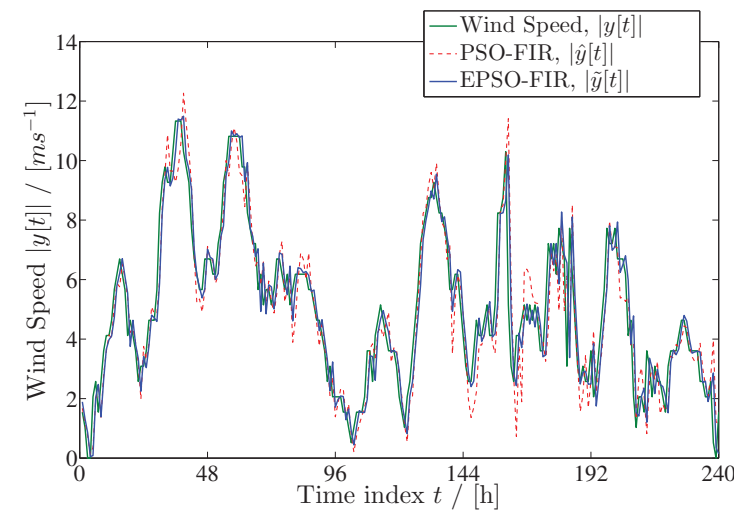

(a) Chivenor

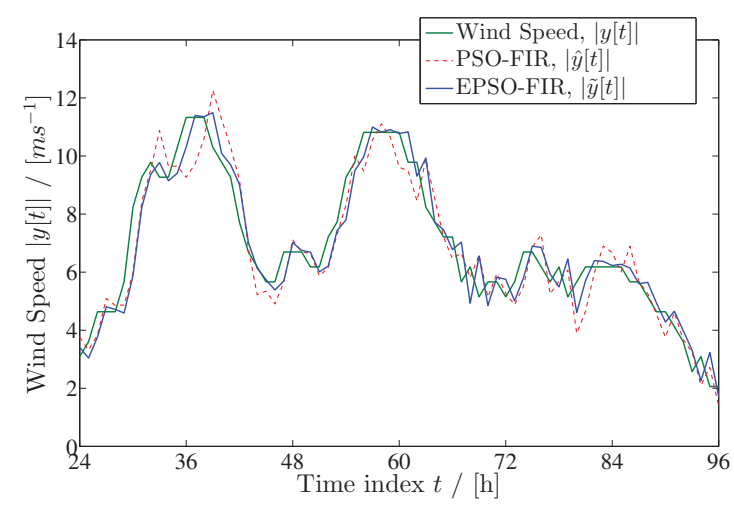

(c) Chivenor

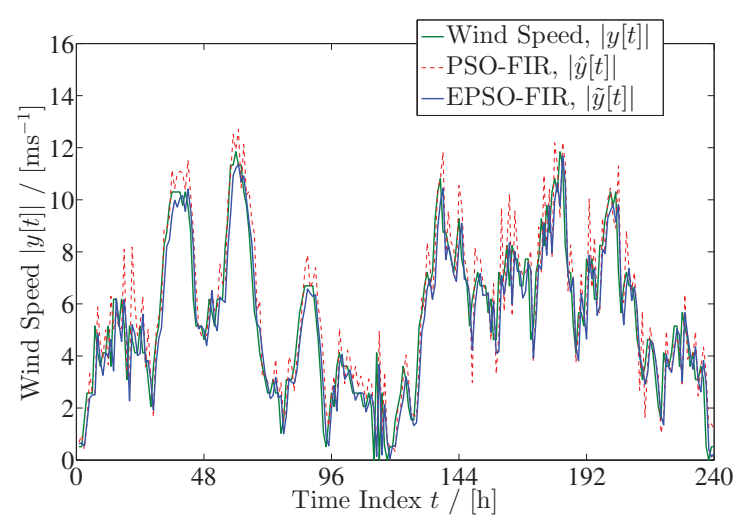

(b) Rhoose

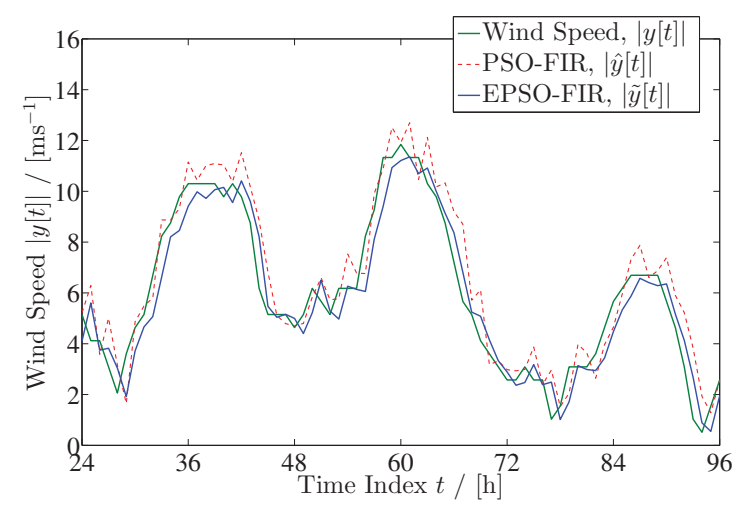

(d) Rhoose

Figure 2. Wind speed, individual PSO-FIR prediction and ensemble prediction (EPSO-FIR) for (a,c) Chivenor and (b,d) Rhoose. (a) and (b) show 31/05/97-09/06/97, while (c) and (d) show 01/06/97-03/06/97.

real part of the first element of the PSO particles is shown in Figure 3. Also of note is that the EPSO-FIR requires very little training data, approximately $2 N$ samples to populate the filter and converge, compared to the CLMS which, depending on choice of learning rate and training strategy requires several months of data, and the CsWF which needs several years worth of training data in order to capture the seasonal trends in the wind data.

\section{Conclusions and Future Work}

The ensemble particle swarm optimised FIR predictor proposed offers similar performance to linear techniques of higher complexity, which require substantially more training data, and still has great potential for further development. The PSO algorithm is found to be efficient and converge quickly, tracking the non-stationary wind signal well.

The potential for tracking large changes in wind speed is of great interest since this is a weakness of the simple linear and many substantially more complex techniques which are currently employed for short-term wind prediction.

The performance of this early-development approach are encouraging and the method warrants further investigation. Both the complex LMS and cyclo-stationary Wiener filter saw significant improvement when expanded to process information from multiple sites simultaneously, taking advantage of the spatial correlation between different locations, $[3,4]$. We expect to see similar results for the ensemble particle swarm optimised FIR filter approach. The EPSO-FIR will also be extended to forecast more than 1 time step ahead.

Other authors have had success combining PSO with multiscale analysis such as wavelet decomposition and other techniques, $[11,12]$. These should be investigated along with other appropriate PSO variations $[6,7,13]$.

\section{Acknowledgements}

The authors gratefully acknowledge the British Atmospheric Data Centre for their supply of meteorological data, and the support of the UK's Engineering and Physical Sciences Research Council via the University of Strathclyde's Wind Energy Systems Centre for Doctoral Training, grant number EP/G037728/1. 


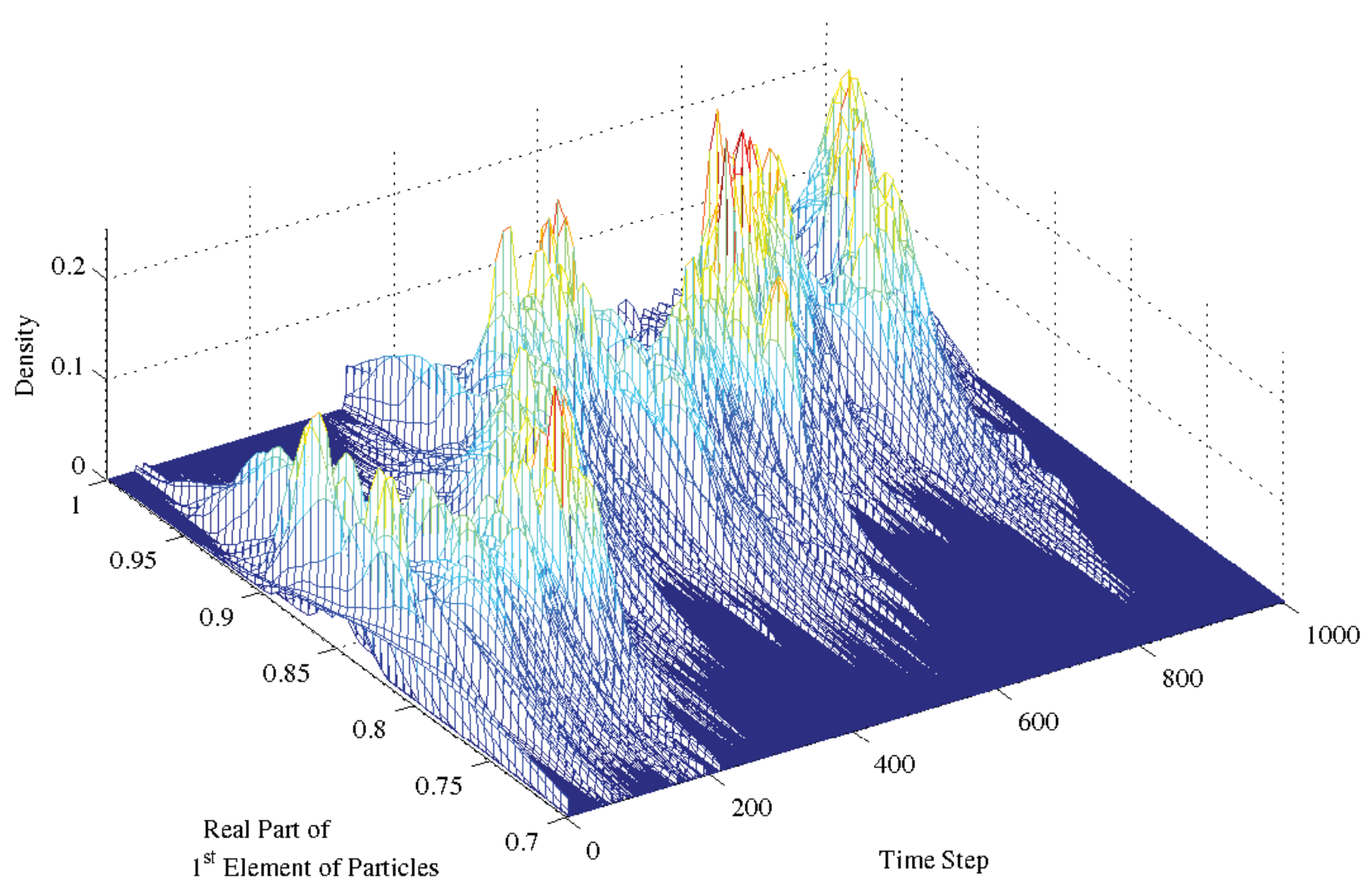

Figure 3. An example of the density evolution of the real part of the first element of the particles in a swarm for the first 1000 time steps of prediction.

\section{References}

[1] G. Giebel, "The state-of-the-art in short-term prediction of wind power — a literature overview," Ris $\varnothing$ National Laboratory, Tech. Rep., 2011.

[2] S. J. Watson, L. Landberg, and J. A. Halliday, "Application of wind speed forecasting to the integration of wind energy into a large scale power system," IEE Proceedings —- Generation, Transmission and Distribution, vol. 141, no. 4, pp. 357-362, 1994.

[3] J. Dowell, S. Weiss, D. Hill, and D. Infield, "Improved spatial modelling of wind fields," in EWEA Annual Conference, Vienna, Austria, Feb. 2013.

[4] J. Dowell, S. Weiss, D. Hill, and D. Infield, "A cyclo-stationary complex multichannel Wiener filter for the prediction of wind speed and direction," in Proceedings of the European Signal and Image Processing Conference, Marrakech, Morocco, Sept. 2013, to appear.

[5] J. Kennedy and R. Eberhart, "Particle swarm optimization," in IEEE International Conference on Neural Networks, vol. 4, pp. 1942-1948, 1995.

[6] R. Eberhart and Y. Shi, "Particle swarm optimization: developments, applications and resources," in Proceedings of the 2001 Congress on Evolutionary Computation, Seoul, Korea vol. 1, pp. 81-86, May 2001.

[7] A. Y. Alanis, C. Simetti, L. J. Ricalde, and F. Odone, "A wind speed neural model with particle swarm optimization kalman learning," in World Automation Congress, pp. 1-5, 2001.

[8] R. Kiran, S. Jetti, and G. Venayagamoorthy, "Online training of a generalized neuron with particle swarm optimization," in
International Joint Conference on Neural Networks, pp. 5088 5095, 2006.

[9] B. Widrow, J. McCool, and M. Ball, "The complex LMS algorithm," Proceedings of the IEEE, vol. 63, no. 4, pp. 719-720, 1975.

[10] [Online]. Available: http://badc.nerc.ac.uk

[11] J. Catalão, H. M. I. Pousinho, and V. Mendes, "Hybrid waveletPSO-ANFIS approach for short-term wind power forecasting in portugal," IEEE Transactions on Sustainable Energy, vol. 2, no. 1 , pp. 50-59, 2011.

[12] W. Xiao-lu, L. Jian, and L. Jian-jun, "Wavelet transform and pso support vector machine based approach for time series forecasting," in International Conference on Artificial Intelligence and Computational Intelligence, vol. 1, pp. 46-50, 2009.

[13] X. Hu, Y. Shi, and R. Eberhart, "Recent advances in particle swarm," in Congress on Evolutionary Computation, vol. 1, pp. 90-97, 2004. 